\title{
Occupational pesticide exposure in early pregnancy associated with sex-specific neurobehavioral deficits in the children at school age
}

\section{Citation}

Andersen, Helle R., Fróđi Debes, Christine Wohlfahrt-Veje, Katsuyuki Murata, and Philippe Grandjean. 2015. "Occupational Pesticide Exposure in Early Pregnancy Associated with SexSpecific Neurobehavioral Deficits in the Children at School Age." Neurotoxicology and Teratology 47 (January): 1-9. doi:10.1016/j.ntt.2014.10.006.

\section{Published Version}

10.1016/j.ntt.2014.10.006

\section{Permanent link}

http://nrs.harvard.edu/urn-3:HUL.InstRepos:37221748

\section{Terms of Use}

This article was downloaded from Harvard University's DASH repository, and is made available under the terms and conditions applicable to Open Access Policy Articles, as set forth at http:// nrs.harvard.edu/urn-3:HUL.InstRepos:dash.current.terms-of-use\#OAP

\section{Share Your Story}

The Harvard community has made this article openly available.

Please share how this access benefits you. Submit a story.

Accessibility 
Occupational pesticide exposure in early pregnancy associated with sex-specific neurobehavioral deficits in the children at school age

Helle R. Andersen ${ }^{1}$, Fróði Debes ${ }^{1}$, Christine Wohlfahrt-Veje ${ }^{2}$, Katsuyuki Murata ${ }^{3}$, Philippe Grandjean $^{1,4}$,

${ }^{1}$ Institute of Public Health, University of Southern Denmark, Odense, Denmark;

${ }^{2}$ University Department of Growth and Reproduction, Rigshospitalet, Copenhagen, Denmark;

${ }^{3}$ Department of Environmental Health Sciences, Akita University Graduate School of Medicine, Akita, Japan;

${ }^{4}$ Harvard School of Public Health, Boston, MA, USA

\section{Corresponding author:}

Helle Raun Andersen, PhD

J.B.Winsløws Vej 17A

DK-5000 Odense C

Mail: hrandersen@health.sdu.dk

Phone: +4565503765 


\begin{abstract}
Prenatal exposure to pesticides may affect neurodevelopment, but the impact of modern pesticides is unclear. During 1997-2001, women working in greenhouse horticultures were recruited in the beginning of their pregnancy. Based on detailed interview of the women and their employers, those categorized as occupationally exposed to pesticides were moved to unexposed work functions or went on paid leave, while women without any exposure were considered unexposed controls. Of the resulting birth cohort of 203 children, 133 (65\%) were examined at age 6-to-11 years together with 44 newly recruited children of same age whose mothers were not occupationally exposed to pesticides in pregnancy. All children underwent a standardized examination including a battery of neurodevelopmental tests. Maternal occupational pesticide exposure in early pregnancy was associated with prolonged brainstem auditory evoked potential latencies in the children as a whole and with impaired neuropsychological function in girls, while no effect was apparent in boys. In girls, language and motor speed functions were significantly inversely associated with prenatal exposure, and a non-significant tendency toward decreased function was also seen for other neuropsychological outcomes. A structural equation model that combined all these test results showed an overall impaired neuropsychological function in girls prenatally exposed to pesticides. Thus, our findings suggest an adverse effect of maternal occupational pesticide exposure on their children's neurodevelopment, despite the fact that the exposures occurred only during early pregnancy and at well regulated working conditions, where special measures to protect pregnant women were applied.
\end{abstract}

\title{
Keywords
}

Pesticides, prenatal exposure, neurodevelopment, neuropsychological tests, brainstem auditory evoked potentials 


\section{Abbreviations}

ADHD: Attention deficit hyperactivity disorder

BAEP: Brainstem auditory evoked potential

CVRR: Coefficient of variation for heart rate

CPT II: Conners' Continuous Performance Test II

SEM: structural equation model

WISC-R: the Wechsler Intelligence Scale for Children-Revised

WJ-III: The Woodcock-Johnson III Test of Cognitive Abilities 


\section{Background}

Prenatal exposures to neurotoxic or neuroendocrine disrupting chemicals are thought to contribute to impaired neurodevelopment and related disorders (Frye et al. 2012; Grandjean and Landrigan 2014). Many pesticides possess neurotoxic and endocrine disrupting properties (Andersen et al. 2002; Bjorling-Poulsen et al. 2008) and experimental and epidemiological research suggests that low-dose prenatal and early postnatal pesticide exposure may adversely affect neurodevelopment (Bjorling-Poulsen et al. 2008). Thus, maternal occupational exposure to mixtures of pesticides during pregnancy has been associated with impaired neurodevelopment (Grandjean et al. 2006; Harari et al. 2010). In prospective birth cohort studies, maternal exposure to chlorpyrifos and other organophosphate insecticides in pregnancy was associated with neurobehavioral deficits in the children that persisted until at least 7 years of age (Bouchard et al. 2011; Eskenazi et al. 2007; Marks et al. 2010; Rauh et al. 2011; Rauh et al. 2006). Additionally, prenatal exposure to chlorpyrifos was associated with structural brain anomalies at school-age including disruption of normal sexual dimorphisms in brain structure (Rauh et al. 2012). Sex-specific effects are in accordance with findings in rodents, where low-dose exposure to chlorpyrifos during brain development, interfered with sex differences in the neurotransmitter system and related cognitive functions and behaviors later in life (Aldridge et al. 2004; Levin et al. 2002; Venerosi et al. 2012). Sex-selective disturbances in brain development after gestational exposure have also been reported in animal studies of other organophosphates (Levin et al. 2010) and for anti-androgenic fungicides (Bisenius et al. 2006; Vinggaard et al. 2005).

To investigate potential health effects of prenatal pesticide exposure, we have established and followed a cohort of children, whose mothers were employed in greenhouse horticulture during pregnancy. Some of the mothers were occupationally exposed to mixtures of modern pesticides with short biological half-lives (hours to few days) during the first trimester of pregnancy, while 
unexposed mothers served as controls (Andersen et al. 2008). A considerable part of the pesticides used have neurotoxic and endocrine disrupting properties (Andersen et al. 2002; Orton et al. 2011). From this cohort, we have previously reported associations between maternal pesticide exposure in pregnancy and lower birth weight followed by higher body fat content at school age (WohlfahrtVeje et al. 2011), impaired reproductive development in boys at 3 months (Andersen et al. 2008) and at school age (Wohlfahrt-Veje et al. 2012a), and earlier breast development in girls (WohlfahrtVeje et al. 2012b). We hypothesized that maternal occupational exposure to pesticides in early pregnancy would be associated with impaired neurodevelopment in the children at school age, possibly in a sex-specific manner. To test the hypothesis, we compared neurodevelopment among children born to mothers who were occupationally exposed to pesticides during early pregnancy to children of unexposed mothers.

\section{Methods}

\subsection{Study population and design}

This study is a part of an ongoing prospective study of the effects of pesticide exposure in early pregnancy on the growth and development in the children. From 1996 to 2000, we recruited pregnant women working in greenhouses and referred to the Department of Occupational Health at Odense University Hospital in Denmark for risk assessment of their working conditions and guidance for safe work practices during pregnancy. Following childbirth, their children were first examined at three months of age (Andersen et al. 2008) and then re-examined at school age (Wohlfahrt-Veje et al. 2011).

Details on this study, including recruitment procedure and exposure assessment, have been described previously (Andersen et al. 2008; Wohlfahrt-Veje et al. 2011). Briefly, detailed 
information about working conditions for the previous three months (i.e., job function, personal handling of pesticides, names and application frequency of pesticide products, reentry intervals for entering greenhouses where pesticides had been applied, procedures for handling of plant cultures recently treated with pesticides, and use of protective equipment) was obtained from maternal interview at enrollment (gestational weeks 4-10) and supplemented by telephone contact to the employers.

For all women, re-entry activities (such as moving or packing potted plants or nipping cuttings) constituted their main work functions. Approximately $20 \%$ of the women reported having been directly involved in applying pesticides, mainly by irrigating fungicides or growth retardants. Only few $(6 \%)$ of the women had applied insecticides. The women were categorized as occupationally exposed if pesticides were applied in the working area more than once a month and the women handled treated plants within one week after treatment and/or the women were directly involved in applying pesticides. The women were categorized as occupationally unexposed if none of the above criteria was fulfilled. All exposure assessments and categorization of the mothers as pesticide exposed or unexposed were performed independently by two toxicologists with special expertise in working conditions in greenhouse horticultures and completed before the first examination of children. Full agreement occurred in all cases. Women categorized as pesticide exposed, went on paid leave or were moved to work functions with less or no pesticide exposure shortly after enrollment. Hence, the exposure classification relates to the early weeks of the first trimester before study enrollment. The median number of days from conception to removal from exposed work functions was 33 days (95\% CI: 15-83).

The exposure situation was complex since the use of specific pesticides varied with time and location, both within the same company and between companies, depending on the plant production 
and the type of pest to be controlled. Approximately 200 different pesticide formulations, representing 124 different active pesticide ingredients, were used in the greenhouses. Some were used only in few greenhouses or during restricted time periods, whereas others were used more often. Several insecticides including organophosphates (dichlorvos, dimethoate, chlorpyrifos), pyrethroids (deltamethrin, fenpropathrin) and carbamates (methiocarb, methomyl) were used frequently in the working areas. However, the time interval between applying insecticides and working in the treated areas was longer (1-3 days) than for fungicides and growth regulators (often a few hours). Hence, the re-entry exposure was regarded higher for fungicides and growth regulators than for insecticides. Given the complexity of the exposure situation, it was not feasible to include the number and type of pesticides in the exposure categorization. A complete list of the pesticides used in the greenhouses can be obtained from the corresponding author.

A total of 168 children were categorized as prenatally exposed to pesticides, and 35 children were categorized as unexposed (because the mothers were employed in administration or similar tasks not involving contact with pesticides). Of these children, $133(65.5 \%)$ accepted an invitation to participate in a follow-up study when the children were between 6 and 11 years old. Within this group, 112 (59 boys and 53 girls) were prenatally pesticide-exposed and 21 (14 boys and 7 girls) were unexposed. To supplement the unexposed group, the participating families were asked to invite children among relatives, friends and neighbors to participate. Only children between 6 and 11 years, whose mother had not been occupationally exposed to pesticides during pregnancy, could participate. By this approach, 44 additional unexposed children were recruited (Wohlfahrt-Veje et al. 2011).

\subsection{Ethics}


The study was conducted according to the Helsinki II Declaration with written informed consent by all mothers as approved by The Regional Scientific Ethical Committee for Southern Denmark and the Danish Data Protection Agency.

\subsection{Questionnaire}

All families completed a questionnaire forwarded prior to the examination of the child with information on medical history, education, occupation, living conditions, life style and diet. An additional short questionnaire regarding smoking, alcohol intake, medicine use, disease and occupation during pregnancy was answered by the 'new' families for whom we did not have this information from the first examination. The pediatrician reviewed the questionnaire responses together with the families (the child and accompanying parents) in connection with the clinical examination. Information on gestational age at birth, head circumference at birth, birth weight and length was obtained from obstetric records.

Social class of the family was based on the parents education and occupation according to the criteria of the Danish National Institute of Social Research (Hansen 1978), which is almost identical to the UK Registrar General's classification of five social classes ranked 1 (high) to 5 (low). We used the social class of the higher ranked parent living with the child.

\subsection{Clinical examination}

The children underwent a standardized physical examination including neurologic functions: social response (interested, disinterested, reluctant, refusing); spontaneous motility (sitting); involuntary movements (posture with arms extended); Romberg's sign (15 sec); walking on a straight line (normal, on tiptoes, on heels, and on the sides of the feet); standing on one leg (seconds); number of 
hops (on one leg, on toes); biceps and patellar reflexes; finger opposition test; diadochokinesis; finger-nose coordination (eyes closed); hearing (otoscopy and audiometry); and vision (spontaneous and directional nystagmus, pupillary reaction to light).

With the child relaxing in a chair, Systolic and diastolic blood pressure was measured three times with an automated non-invasive blood pressure monitor (Colin Press-Mate, Colin ${ }^{\circledR}$ Corporation, Japan) and means were used for data-analysis. Body weight $(\mathrm{kg})$ was measured on a digital weight scale with a precision of $0.1 \mathrm{~kg}(\mathrm{TBF}-300$, Tanita Europe, UK). Standing height $(\mathrm{cm})$ was measured to the nearest $0.1 \mathrm{~cm}$ using a transportable stadiometer (Chasmors LTD., London, UK). The body mass index (BMI) was calculated as the weight (in $\mathrm{kg}$ ) divided by the height (in $\mathrm{m}$ ) squared.

\subsection{Neurophysiological tests}

As a measure of autonomic nervous system function, heart rate variation was measured using a Heart Rate Monitor (Polar s810) connected to a computer under standardized conditions as previously described (Grandjean et al. 2004). Briefly, after the child had been lying in a relaxed, supine position for at least 5 minutes, 300 R-R (beat to beat) intervals were measured and the 100 consecutive R-R intervals with the lowest standard deviation (SD) were extracted. We calculated the coefficient of variation for heart rate $\left(\mathrm{CV}_{\mathrm{RR}}\right)$ as the ratio in (percent) of the $\mathrm{SD}$ to the mean of the R-R interval. Short-term variations in heart rate reflect normal homeostatic mechanisms of cardiac autonomic activity and adverse effects seem mainly to result in a relative depression of parasympathetic activity (Araki et al. 1997). 
With the child placed comfortably in a chair, brainstem auditory evoked potential (BAEP) latencies were recorded using a four-channel electromyography (Neuropack MEB-9104, Nihon Kohden, Japan) as previously described (Murata et al. 2004). Click signals at an intensity of $65 \mathrm{~dB}$ hearing level (0.1-msec impulses of alternating polarity) were presented to the right ear through shielded ear phones at rates of $20 \mathrm{~Hz} / \mathrm{sec}$ and $40 \mathrm{~Hz} / \mathrm{sec}$ independently (sampling time, $0.01 \mathrm{msec}$ ). The left ear was masked with white noise at an intensity of $45 \mathrm{~dB}$ HL. BAEP latencies were recorded by using three standard electroencephalogram electrodes placed on the vertex, the right mastoid ipsilateral to the stimulation, and the left mastoid (ground). Peaks I, III, and V are thought to reflect the volumeconducted electric activity from the acoustic nerve, pons (superior olivary nucleus), and midbrain (inferior colliculi), respectively (Murata et al. 2004).

\subsection{Neuropsychological tests}

The time schedule for testing was set to maximum one hour and the composition of tests was chosen to examine a broad spectrum of functions with high psychometrical validity and sensitivity to neurotoxicity of environmental pollutants (Debes et al. 2006; Harari et al. 2010; White et al. 1994).

\subsubsection{Manual motor speed}

In the Finger Tapping test, the child tapped a key for a series of 10 -sec sessions, first completing one session with the preferred hand and one with the non-preferred hand for practice, then alternately two sessions with the preferred and non-preferred hand. We used the standard board for this test (WW-1597-NP; Psychological Assessment Resources, Odessa, FL, USA), but the thickness of the board was increased by $1 \mathrm{~cm}$ to allow children with small hands to move the tapping arm 
effortlessly (Lezak 1995). Means of the number of taps from the non-practice trials for the preferred hand, non-preferred hand, and both hands were used for data-analysis.

\subsubsection{Simple reaction time}

Simple reaction time was assessed by the Catsys ${ }^{\circledR}$ equipment (Danish Product Development, 3070 Snekkersten, Denmark). During sessions of 60 seconds, the child pushed a sensor button on a response rod as fast as possible after an auditory stimulus, first one session with the preferred hand and then a session with the non-preferred hand. Means for dominant and non-dominant hand were used for data-analysis.

\subsubsection{Attention}

In Conners' Continuous Performance Test II (CPT II, version 5 for windows; Multi-Health Systems Inc., North Tonawanda, NY, USA), the child was required to press the spacebar (hits) each time a letter appeared, unless the letter was a X, during a 15-min test duration. Scores derived from the test were the total number of missed responses (omissions), false positives (commissions), and the overall average reaction time.

\subsubsection{Short-term memory}

On the Wechsler Intelligence Scale for Children-Revised (WISC-R) (Wechsler 1974), the Digit Span Test (forward and backward) required that the child repeat strings of digits forward and in reversed order until the child failed both trials. Score was total number of correct trials. The Stanford-Binet IV Memory for Sentences test provides an assessment of auditory span in a naturalistic context of connected speech (Thorndike and Hagen 1986). 


\subsubsection{Language functions and long-term memory}

The Woodcock-Johnson III Tests of Cognitive Abilities (WJ-III) Verbal Comprehension test comprises four subtests: Picture Vocabulary, Synonyms, Antonyms, and Verbal Analogies. In the WJ-III Picture Vocabulary test the child was asked to recognize and name pictures of familiar to less familiar objects. The test was administered in accordance with the Boston Naming Test, which means that a stimulating sound (stimulus cue) was given, if the child did not know what an object represents and the first sound (phonemic cue), if the child could not find the right word. If the child could still not answer, the name of the object was given and the child was then asked whether the name was a word that the child knew. In the synonyms and antonyms subtests, the child was asked for words having the same or opposite meaning when orally presented with a stimulus word. In the Verbal Analogies subtest, the child's word knowledge and ability to reason was tested by requiring the child to provide words that completed orally presented analogies with increasingly complex relationships among words. The four different tasks were scored separately and based on these scores an overall verbal comprehension factor score was calculated. A memory condition was added to the Picture Vocabulary test, where the number of pictures remembered after approx. 60 minutes was recorded. There was no requirement for proper designation.

\subsubsection{Visuospatial performance and memory functions}

Raven's Colored Progressive Matrices (Raven et al. 1979) provide a nonverbal assessment of general intelligence in children 5-11 years of age. The test assesses the ability to detect an organizing principle in visual materials, requiring the examinee to recognize spatial, design, and numerical relationships. 
In the Copying Test of the Stanford-Binet, 4th edition (Thorndike and Hagen 1986), the child copied visual designs by drawing. We administered the second part of the test only (design 13-28). Each design was presented to the child, one at a time, at increasing degree of difficulty. The child was required to copy each design in freehand drawing. The drawings were scored for correct designs after completion of the examination. A memory condition was added where the number of designs remembered after 20 min was recorded.

The neuropsychological tests were administered in a uniform sequence by the same neuropsychologist (FD) who also performed all test scorings. The same pediatrician (CWV) performed all clinical examinations and calculations of all BAEP latencies and heart rate variability were conducted by the same examiner (KM). In all cases the examiners were blinded to information about maternal pesticide exposure during pregnancy and other clinical outcomes.

\subsection{Data analysis}

For all outcomes, age at examination and $\operatorname{sex}(0=$ male, $1=$ female $)$ were considered obligatory covariates. Other potential confounders were selected from a priori considerations of factors that might influence the specific outcome. Maternal ethnicity (Danish or non-Danish origin), smoking and maternal alcohol consumption in pregnancy were treated as a dichotomous variables. Due to the small sample size, social class was re-coded into three categories: groups 1-3, group 4 or group 5 and included in the models as two dummy variables with group 4 as reference as most families belonged to this group.

Differences in characteristics between unexposed and exposed children $(0=$ unexposed, $1=$ exposed) were tested by Mann-Whitney U test (continuous data) or Fisher's exact test (categorical 
data with two categories) or Likelihood Ratio (categorical data with more than two categories). We employed multiple linear regression analyses to examine the associations of prenatal pesticide exposure with neurobehavioral and neuropsychological outcomes.

For analysis of blood pressure and heart rate variability, maternal smoking and alcohol consumption in pregnancy were included as covariates, while child BMI was included in the model for blood pressure. For BAEP latencies, the variable 'diagnosed neurological disease' $(\mathrm{N}=7)$, consistently changed the estimate by more than $10 \%$ and in the same direction. Therefore this variable was included as an independent variable along with age, sex, and impaired hearing. Maternal smoking and alcohol consumption in pregnancy, social class, history of concussion, and impaired vision were considered but were not included in the final equations because of minimal effect on the estimates.

For all neuropsychological outcomes, social class, history of concussion, diagnosed neurological disease, and maternal alcohol intake in pregnancy were included as obligatory covariates. Maternal non-Danish ethnicity was included in outcomes involving language function. Maternal smoking in pregnancy, but none of the other potential confounders, was significantly correlated with social class (Chi-square, $p=0.002)$ and was therefore not considered mandatory. Potential interaction between prenatal pesticide exposure and sex was investigated by including a product term of these two variables in the models. Considerably more of the unexposed control children recruited at follow-up belonged to social class 1-3 compared to children from the original birth cohort (54.5\% vs $18.8 \%$ ). Therefore, sensitivity analyses of neuropsychological outcomes were performed with only unexposed controls from the original birth cohort included. 
To complement the regression analyses of individual psychometric test results, structural equation models (SEMs) were developed to comprise (1) a bi-factor confirmatory factor analytic measurement model specifying a latent variable for general mental ability directly reflected in all the manifest test variables, and six residualized broad latent cognitive abilities each reflected in a number of manifest test variables as indicators of that particular intellectual, perceptual or response modality; (2) a higher-order confirmatory factor analytic measurement model specifying a secondorder latent variable for general mental ability reflected in six first-order broad latent cognitive abilities each again reflected in a number of manifest test variables as indicators of that particular intellectual, perceptual or response modality; (3) two structural models (a) one specifying the changes in the estimated latent variable for general mental ability, commonly referred to as the gfactor, designating a theoretical construct of general intelligence, and (b) another specifying the changes in both the g-factor and residualized the broad ability factors in the bi-factor model. The covariates were the same as in the multiple regression models for the individual tests. Consistent with modern psychometric modeling (Gignac and Watkins 2013; Valerius and Sparfeldt 2014) the first-order broad ability factors were specified as: verbal comprehension (Gc, crystalized intelligence), visual-spatial processing (Gv), short-term memory (Gsm), long-term storage and retrieval (Glr), attention (Gt, reaction and decision speed, chronometrically measured), and psychomotor speed (Gps).

The model provides estimates of the strength of the linear relations between the different components together with goodness-of-fit indices e.g., Chi-Square Test of Model Fit, Root Mean Square Error of Approximation (RMSEA), Comparative fir Index (CFI) and Tucker-Lewis Index (TLI), Standardized Root Mean Square Residual (SRMR), that indicate the adequacy of the model. Specifically the effect of the interaction between sex and prenatal pesticide exposure on the g-factor 
was tested as the main relation of interest. Additionally, the effects on the six first-order factors were tested individually. As the tests selected for inclusion in this study may not fully represent the ability factors of interest, some also being narrowly defined by splitting tests into two scales of even and uneven items, and because the validity of the general ability modeled is not fully known, interpretation of the SEMs must be cautious. However, the statistical advantage of adjusting for imprecision in the exposure assessment and for multiple comparisons supports the use of this approach as a complement to the series of standard regression analyses carried out.

For most statistical analyses, a p-value $\leq 0.05$ was used to determine significance. Because of the lower power to detect an interaction effect (Brookes et al. 2004), a p-value $\leq 0.1$ was considered statistically significant for analyses of interaction effects of sex and prenatal pesticide exposure. 


\section{Results}

A higher fraction of the unexposed children was from social class 1-3 (Table 1), mainly because 24 $(54.4 \%)$ of the newly recruited controls were from social class $1-3$ compared to only $9.5 \%$ of the previously recruited unexposed children and $20.5 \%$ of the exposed children from the original cohort. More unexposed children had a history of concussion. None of the other characteristics deviated significantly between prenatally pesticide-exposed and unexposed children.

Due to technical problems, heart rate measurements and BAEP latencies were performed only for 128 and 90 children, respectively, thus introducing some differences between the children with available data and those without. Relatively more of the children with available heart rate measurements were prenatally exposed $(71.1 \%)$ and fewer were from social class 1-3 (18\%) compared to children with no measurements (43\% exposed and $53 \%$ from social class $1-3$ ). For children with available BAEP latencies, $50 \%$ were prenatally exposed versus $77 \%$ of those without BAEP determinations. Relatively more children with BAEP measurements (35.6\%) were from social class 1-3 compared to those without measurements (19.5\%). However, including social class covariates in the regression analysis for heart rate variability and BAEP latencies did not affect the results obtained. Prenatal pesticide exposure was related to longer brainstem evoked potential latencies (Table 2). Both before and after adjusting for relevant confounders, peak III and V were significantly prolonged at both frequencies of 20 and $40 \mathrm{~Hz}$ (only data for $40 \mathrm{~Hz}$ are presented, as the results for the two frequencies were highly correlated). At $40 \mathrm{~Hz}$, I-III and I-V, but not III-V, inter-peak latencies were prolonged in exposed children. After adjustment, the prolongation for the I-III inter-peak latency was only of borderline significance. Boys had significantly longer latencies for most peaks than girls (results not shown) and prolongation in latencies were more strongly 
correlated to prenatal pesticide-exposure among boys than among girls but a sex-exposure interaction, of borderline significance, was seen only for peak $\mathrm{V}$ and for inter-peak I-V latencies. No associations were found between prenatal pesticide exposure and heart rate variability or blood pressure at school age (results not shown).

The neuropsychological tests were completed by most of the 177 children (Table 3). For the cohort as a whole, there were no significant associations between prenatal pesticide exposure and test results. However, significant interaction $(\mathrm{p} \leq 0.1)$ between sex and prenatal pesticide exposure was found for language functions (picture vocabulary and verbal comprehension factor) and (of borderline significance) for motor speed function (finger tapping test) and auditory short-term memory (Stanford-Binet sentence repetition). For girls, but not boys, prenatal pesticide exposure seem associated with poorer scores in most of the tests although the differences between prenatally pesticide-exposed and unexposed girls reached significance only for the picture vocabulary and finger tapping (both hands) tests (Table A1).

The SEM analysis with a bi-factor measurement model confirmed a significant sex-exposure interaction on general intellectual ability (standardized estimate for exposed girls: $-0.674,95 \%$ CI: 1.066; $-0.282, \mathrm{P}=0.005$ (Figure 1). The model fit was good with Chi Square $=448.996 ; \mathrm{DF}=313$; $\mathrm{P}=0.0000 ; \mathrm{RMSEA}=0.05 ; 90 \% \mathrm{CI}: 0.039 ; 0.06$; Probability RMSEA $\leq 0.05=0.492 ; \mathrm{CFI}=$ 0.900; TLI $=0.885 ; \mathrm{SRMR}=0.073$. An alternative SEM-analysis with a higher-order measurement model also confirmed a significant sex-exposure interaction of the same order of magnitude on general intellectual ability (standardized estimate for exposed girls: $-0.661,95 \%$ CI: $-1.064 ;-0.258$, $\mathrm{P}=0.007$. Again, the model fit was good with Chi Square $=446.549, \mathrm{DF} 318, \mathrm{P}=0.0000 ;$ RMSEA 0.048, $90 \%$ CI: 0.037; 0.058, Probability RMSEA $\leq 0.05=0.603, \mathrm{CFI}=0.906, \mathrm{TLI}=0.89 . \mathrm{SRMR}$ 
$=0.072$. The similarity of the negative sex-exposure effect on the general ability factor across the two types of models attests to the stability and consistency of the g-factor estimate. Separate models for each of the latent first-order factors in the model supported negative effects of sex-exposure interaction for all six abilities but were significant only for verbal comprehension $(\mathrm{Gc}=-0.66,95 \%$ CI: - 1.14; $-0.18, \mathrm{P}=0.02)$, manual motor speed $(\mathrm{Gps}=-0.60,95 \% \mathrm{CI}:-1.12 ;-0.08, \mathrm{p}=0.06)$ and short-term memory $(\mathrm{Gsm}=-0.55,95 \% \mathrm{CI}$ - $1.09 ;-0.02, \mathrm{P}=0.09)$. A comprehensive oblique firstorder model supported this pattern with a significant negative effect on $\mathrm{Gc}(-0.65, \mathrm{P}=0.023)$ and near-significant negative effects on Gsm $(-0.56, \mathrm{P}=0.08)$ and $\mathrm{Gps}(-0.61, \mathrm{P}=0.06)$.

For unexposed children, girls tended to have lower motor speed and longer reaction time than boys. In contrast, unexposed girls tended to score higher in tests of language and visual performance and memory than unexposed boys although the difference was statistically significant only for the Stanford-Binet Copying test $(\mathrm{p}=0.007)$ and visual memory tests $(\mathrm{p}=0.007$ for Stanford-Binet, Copying recall and $p=0.02$ for WJ-III, Picture Naming, recall). Higher scores among girls for these functions were not apparent among prenatally pesticide exposed children. In fact, exposed girls scored lower in test of language, visual performance, and memory than exposed and unexposed boys.

All neuropsychological test outcomes were significantly affected by the age of the child. For example, the regression coefficient for age (in years) for the Verbal comprehension factor was 0.37. The regression coefficient for prenatally pesticide exposed girls was -0.31 (Table A1) equaling a developmental delay in language function corresponding to approximately $0.31 / 0.37=0.8$ years $(10$ months) in girls. 
Social class was considered an important determinant in all the tests. Since more of the newly recruited control children belonged to higher social classes, the sex-exposure interaction regression analyses of individual psychometric test results were repeated with only unexposed controls from the original cohort included. This change did not appreciably change the magnitude or direction of the regression coefficients for most of the test results (Table A2) and, for language function, a significant interaction between sex and pesticide exposure remained.

\section{Discussion}

In this study, maternal occupational pesticide exposure in early pregnancy was associated with prolonged BAEP latencies in the children and, in the girls, with impaired neurobehavioral function. The results indicate an adverse effect on general intellectual development in girls, with most pronounced effects in domains of language function and to a lesser extent in motor speed and shortterm memory functions. No exposure-related effect on neuropsychological function was apparent in the boys.

Several previous studies have demonstrated associations between maternal exposure to pesticides, mainly organophosphates, in pregnancy and impaired neurodevelopment in children. Reported effects in school-age children include impaired motor skills, attention problems, poorer scores for working memory, language function, visuospatial performance, perceptual reasoning, and full-scale IQ (Bouchard et al. 2011; Harari et al. 2010; Rauh et al. 2011). Only a few studies have investigated if exposure-related effects differed between boys and girls. Maternal urinary excretion of organophosphate metabolites in pregnancy was associated with poorer attention and higher attention deficit hyperactivity disorder (ADHD) scores among boys at 3.5 years and 5 years, but not 
in girls (Marks et al. 2010). In another study, prenatal exposure to the organophosphate chlorpyrifos, evaluated from umbilical cord blood concentration at delivery, was associated with greater decrement in working memory in boys than in girls at age 7 (Horton et al. 2012). In a recent study, residential use of pyrethroid insecticides during pregnancy was associated with greater decrement in psychomotor development in girls than in boys at 14 months (Llop et al. 2013) in agreement with our findings of more pronounced neurodevelopmental effects in girls. Unexposed girls performed better than boys in most of the tests but this sex-difference was apparently reduced or inverted in prenatally exposed children. Differences in sex-specific susceptibility between the studies might be related to differences in exposures. In our study, the mothers were occupationally exposed to mixtures of different pesticides including some organophosphates and other insecticides as well as a preponderance of fungicides and growth regulators (Andersen et al. 2008) with an endocrine disrupting potential (Andersen et al. 2002; Orton et al. 2011). Endocrine disrupting substances that interfere with sex hormone receptors or steroidogenesis might alter the sex dimorphism in brain development and structure, one effect being that neurobehavioural differences between males and females are lessened (Venerosi et al. 2012; Weiss 2011). Interestingly, when brain morphology was investigated by magnetic resonance imaging in a subgroup of the children prenatally exposed to chlorpyrifos, mentioned above (Horton et al. 2012), children with high prenatal exposure did not show the expected sexual dimorphisms in brain morphology as seen in children with low exposure (Rauh et al. 2012). This observation is in accordance with findings in rodents, where low-dose exposure to chlorpyrifos during brain development interfered with hypothalamic neuroendocrine pathways and sex-dependent differences in cognitive functions and behaviors later in life (Aldridge et al. 2004; Levin et al. 2002; Venerosi et al. 2012). 
Human brain development begins early in prenatal life and continues during childhood and adolescence. The growth and functional development is not uniform but follows sexually dimorphic trajectories promoted by sex hormones in complex interaction with genetic and epigenetic factors (McCarthy and Nugent 2013). Sexual differentiation of the brain is initiated by the action of sex-hormones produced following fetal gonadal differentiation which occurs in gestational week 7-8 in humans. From this period, brain development is likely vulnerable to endocrine disrupting substances although the critical windows are incompletely known and may differ between the two sexes. Animal studies indicate that the sex-specific cellular response to the same sex hormones are different in specific brain regions and involve different molecular pathways. For example, estradiol does not seem to masculinize males and feminize females at the same site and time (Bakker and Brock 2010). Thus, only one sex may be sensitive to a specific exposure for a specific outcome. In animal studies, sex-selective disturbances in brain development and behavior have been reported for several pesticides including some organophosphates (Levin et al. 2010), endosulfan (Lafuente and Pereiro 2013), prochloraz (Vinggaard et al. 2005), and mixture of fungicides (Jacobsen et al. 2012). In humans, sexually dimorphic response profiles have been reported in relation to prenatal exposure to endocrine disruptors. For example, bisphenol A was associated with externalizing behavior in girls at two years of age but not in boys (Braun et al. 2009), phthalates were associated with reduced masculine play behavior in boys but had no effect in girls (Swan et al. 2010), and PCBs were associated with feminization of play behavior in boys and masculinization of play behavior in girls (Braun et al. 2009; Winneke et al. 2014).

Disturbances of hormone mediated processes other than those related to steroid hormones may occur, as thyroid hormones and glucocorticoids are important for normal brain development as well (Schantz and Widholm 2001). Dithiocarbamate fungicides, which were used in some of the 
greenhouses included in this study, have been identified as disruptors of both thyroid hormone (Marinovich et al. 1997) and glucocorticoid action (Atanasov et al. 2003). Thus, pesticides have the potential to interfere with several neuroendocrine signal pathways affecting sex-dimorphic brain development as well as hypothalamic regulation of reproduction, metabolism, appetite, and blood pressure (Panzica et al. 2011; Rice and Barone 2000). Accordingly, associations between prenatal pesticide exposure and impaired reproductive development in boys, earlier breast development in girls, increased body fat accumulation from birth to school age accompanied by changes in reproductive hormones and adipokines were also seen in our cohort (Andersen et al. 2008; Andersen et al. 2012; Wohlfahrt-Veje et al. 2012a; Wohlfahrt-Veje et al. 2012b; Wohlfahrt-Veje et al. 2011).

Furthermore, neurotoxic pesticides like organophosphates and pyrethroids may directly disturb neural developmental processes such as neurogenesis, proliferation, migration, synaptogenesis, apoptosis, and myelination by disturbing DNA-synthesis or by interfering with neurotransmitters with neurotrophic action such as acetylcholine (Rice and Barone 2000; Slotkin 2004). Such perturbations may affect neurophysiological processing. The observed association between prenatal pesticide exposure and prolonged BAEP latencies for peaks III and V and the I-III and I-V interpeak intervals are very similar to effects reported after prenatal exposure to the well-known developmental neurotoxicant methylmercury in Faroese children at ages 7 and 14 (Grandjean et al. 1997; Murata et al. 2004). However, in Ecuadorian children exposed prenatally to pesticides, no effects on BAEP latencies were seen (Harari et al. 2010). In contrast to the neuropsychological test outcomes, BAEP latencies are considered to be independent of socioeconomic covariates (Murata et al. 2004) as also confirmed in our study. Likewise, no sex-specific pattern was apparent, but the ability to detect such an association is hampered by the lower sample size $(\mathrm{N}=90)$ for this 
examination. Another explanation could be that effects on BAEPs are due to other pesticides than those affecting neurobehavioral functions.

Our study cannot identify individual pesticides related to our findings, since the study design did not allow bio-monitoring of pesticide exposure in the mothers, and the exposure classification encompassed more than 100 pesticides used in different mixtures (Andersen et al. 2008). Although this is a limitation of our study, the existence of a mixed exposure is a real-world situation, and the blinded exposure classification, the blinded clinical examinations, and the longitudinal design minimized the possible impact of exposure misclassification and bias.

The small sample size results in limited statistical power to detect subtle associations between prenatal pesticide exposure and neurobehavioral performance. To gain statistical power additional unexposed children were recruited at school age. However, this led to small differences in social class between exposed and unexposed children and thereby introducing possible confounding. Social class, as based on parent's education and occupation, is known to be important for neurobehavioral function of the children. We therefore adjusted for this confounder in our statistical analyses, and this adjustment did not affect the observed pesticide-related associations. Besides, we repeated the regression analyses for all neuropsychological outcomes after excluding the newly recruited control children and thereby eliminating the difference in social class between exposed and unexposed children As expected, the confidence intervals were broader, but the general direction of the regression coefficients was unchanged, thus supporting an overall adverse effect in girls with significant sex-exposure interaction for language function. Finally, confounding by social class would be expected to affect girls and boys equally and therefore cannot explain the sexspecific associations identified. 
More than $30 \%$ of the children from the original birth cohort were lost to follow-up at school age, and this attrition introduces a possibility for selection bias. Mothers knowing they had been exposed to pesticides may be more inclined to stay in the study, and parents of children with behavioral problems or learning disabilities in school could perhaps be more motivated to having their children tested. For newly included control children, such selection bias would, however, reduce the ability to detect an exposure-related effect.

Another limitation is that many statistical analyses and multiple comparisons were carried out which may increase the likelihood of spurious associations. However, several of the neuropsychological tests are interrelated and the consistent findings of adverse effects in girls, but not in boys, supported by the joint SEM-analysis suggest a true association between prenatal exposure and impaired neurodevelopment in girls. An advantage of using SEM is that the problem of multiple testing is avoided, since only one statistical test is performed at a time. In addition, the true variance is extracted from all the psychometric tests, while error variance is cancelled out. In support of the SEMs applied, the general factor is well predicted by relevant variables, such as age, social status, and neurological disease. In agreement with empirical findings (Valerius and Sparfeldt 2014) in regard to g-factors extracted from different intelligence test batteries being very highly correlated, the present study adds some optimism regarding the validity of the $g$-factor modeled. The frequent finding of superior predictive validity of measures of general intelligence in the literature emphasizes the importance of examining the effect of neurotoxic substances on estimates of this measure. Thus, the overall difference in general ability suggests that pesticide exposure during early gestation may affect cognition development in general terms, and the present study should therefore inspire further examination of this possibility. 
In conclusion, the results indicate that occupational exposure to mixtures of non-persistent pesticides early in pregnancy, before recognition of the pregnancy and introduction of preventive measures, may affect neurodevelopment in the children. Individual culprits cannot be identified, but the study reflects the common situation of mixed exposures under occupational circumstances, where exposures are properly controlled according to stringent regulations. Although modern pesticides used in greenhouses are thought to break down within few days, it is possible that small residues may linger in the blood stream or the placenta for several days. Also, any endocrine changes caused by the pesticides may normalize with a delay. Accordingly, the main impact on fetal brain development must have happened during the first trimester. The global cognitive deficits in the girls along with the delayed evoked potentials in both girls and boys would be in accordance with a neurotoxic effect during early brain development. Although our study has several limitations and we cannot entirely exclude residual confounding or chance, the associations may be important if detectable in a small study like ours. Given the serious implications of developmental neurotoxicity of the magnitude observed in the present study, improved measures for protection of workers, especially young women, against pesticide exposure in occupational settings seem warranted.

\section{Acknowledgements}

The study was supported by grants from the Danish Environmental Protection Agency. We are grateful to the children and families for their participation in the study. We thank Lene Barfod and Mariann Bøllund and the study team for the skilled help with child examinations and the database. 


\section{References}

Aldridge, J.E.; Seidler, F.J.; Slotkin, T.A. Developmental exposure to chlorpyrifos elicits sexselective alterations of serotonergic synaptic function in adulthood: critical periods and regional selectivity for effects on the serotonin transporter, receptor subtypes, and cell signaling. Environ Health Perspect. 112:148-155; 2004

Andersen, H.R.; Schmidt, I.M.; Grandjean, P.; Jensen, T.K.; Budtz-Jorgensen, E.; Kjaerstad, M.B.; Baelum, J.; Nielsen, J.B.; Skakkebaek, N.E.; Main, K.M. Impaired reproductive development in sons of women occupationally exposed to pesticides during pregnancy. Environ Health Perspect. 116:566-572; 2008

Andersen, H.R.; Vinggaard, A.M.; Rasmussen, T.H.; Gjermandsen, I.M.; Bonefeld-Jorgensen, E.C. Effects of currently used pesticides in assays for estrogenicity, androgenicity, and aromatase activity in vitro. Toxicology and applied pharmacology. 179:1-12;2002

Andersen, H.R.; Wohlfahrt-Veje, C.; Dalgard, C.; Christiansen, L.; Main, K.M.; Nellemann, C.; Murata, K.; Jensen, T.K.; Skakkebaek, N.E.; Grandjean, P. Paraoxonase 1 polymorphism and prenatal pesticide exposure associated with adverse cardiovascular risk profiles at school age. PLoS One. 7:e36830; 2012

Araki, S.; Yokoyama, K.; Murata, K. Neurophysiological methods in occupational and environmental health: methodology and recent findings. Environ Res. 73:42-51; 1997

Atanasov, A.G.; Tam, S.; Rocken, J.M.; Baker, M.E.; Odermatt, A. Inhibition of 11 betahydroxysteroid dehydrogenase type 2 by dithiocarbamates. Biochem Biophys Res Commun. 308:257-262; 2003

Bakker, J.; Brock, O. Early oestrogens in shaping reproductive networks: evidence for a potential organisational role of oestradiol in female brain development. Journal of neuroendocrinology. 22:728-735; 2010

Bisenius, E.S.; Veeramachaneni, D.N.; Sammonds, G.E.; Tobet, S. Sex differences and the development of the rabbit brain: effects of vinclozolin. Biol Reprod. 75:469-476; 2006

Bjorling-Poulsen, M.; Andersen, H.R.; Grandjean, P. Potential developmental neurotoxicity of pesticides used in Europe. Environ Health. 7:50; 2008

Bouchard, M.F.; Chevrier, J.; Harley, K.G.; Kogut, K.; Vedar, M.; Calderon, N.; Trujillo, C.; Johnson, C.; Bradman, A.; Barr, D.B.; Eskenazi, B. Prenatal exposure to organophosphate pesticides and IQ in 7-year-old children. Environ Health Perspect. 119:1189-1195; 2011

Braun, J.M.; Yolton, K.; Dietrich, K.N.; Hornung, R.; Ye, X.; Calafat, A.M.; Lanphear, B.P. Prenatal bisphenol A exposure and early childhood behavior. Environ Health Perspect. 117:1945-1952; 2009

Brookes, S.T.; Whitely, E.; Egger, M.; Smith, G.D.; Mulheran, P.A.; Peters, T.J. Subgroup analyses in randomized trials: risks of subgroup-specific analyses; power and sample size for the interaction test. Journal of clinical epidemiology. 57:229-236; 2004

Debes, F.; Budtz-Jorgensen, E.; Weihe, P.; White, R.F.; Grandjean, P. Impact of prenatal methylmercury exposure on neurobehavioral function at age 14 years. Neurotoxicol Teratol. 28:536-547; 2006

Eskenazi, B.; Marks, A.R.; Bradman, A.; Harley, K.; Barr, D.B.; Johnson, C.; Morga, N.; Jewell, N.P. Organophosphate pesticide exposure and neurodevelopment in young MexicanAmerican children. Environ Health Perspect. 115:792-798; 2007

Frye, C.A.; Bo, E.; Calamandrei, G.; Calza, L.; Dessi-Fulgheri, F.; Fernandez, M.; Fusani, L.; Kah, O.; Kajta, M.; Le Page, Y.; Patisaul, H.B.; Venerosi, A.; Wojtowicz, A.K.; Panzica, G.C. 
Endocrine disrupters: a review of some sources, effects, and mechanisms of actions on behaviour and neuroendocrine systems. Journal of neuroendocrinology. 24:144-159; 2012

Gignac, G.E.; Watkins, M.W. Bifactor modeling and the estimation of model-based reliability in the WAIS-IV. Multivariate Behavioral Research. 48:639-662; 2013

Grandjean, P.; Harari, R.; Barr, D.B.; Debes, F. Pesticide exposure and stunting as independent predictors of neurobehavioral deficits in Ecuadorian school children. Pediatrics. 117:e546$556 ; 2006$

Grandjean, P.; Landrigan, P.J. Neurobehavioural effects of developmental toxicity. Lancet neurology. 13:330-338; 2014

Grandjean, P.; Murata, K.; Budtz-Jorgensen, E.; Weihe, P. Cardiac autonomic activity in methylmercury neurotoxicity: 14-year follow-up of a Faroese birth cohort. The Journal of pediatrics. 144:169-176; 2004

Grandjean, P.; Weihe, P.; White, R.F.; Debes, F.; Araki, S.; Yokoyama, K.; Murata, K.; Sorensen, N.; Dahl, R.; Jorgensen, P.J. Cognitive deficit in 7-year-old children with prenatal exposure to methylmercury. Neurotoxicology and teratology. 19:417-428; 1997

Hansen, E.J. Distribution of living conditions. Copenhagen: Danish National Institute of Social Research; 1978

Harari, R.; Julvez, J.; Murata, K.; Barr, D.; Bellinger, D.C.; Debes, F.; Grandjean, P. Neurobehavioral deficits and increased blood pressure in school-age children prenatally exposed to pesticides. Environ Health Perspect. 118:890-896; 2010

Horton, M.K.; Kahn, L.G.; Perera, F.; Barr, D.B.; Rauh, V. Does the home environment and the sex of the child modify the adverse effects of prenatal exposure to chlorpyrifos on child working memory? Neurotoxicol Teratol. 34:534-541; 2012

Jacobsen, P.R.; Axelstad, M.; Boberg, J.; Isling, L.K.; Christiansen, S.; Mandrup, K.R.; Berthelsen, L.O.; Vinggaard, A.M.; Hass, U. Persistent developmental toxicity in rat offspring after low dose exposure to a mixture of endocrine disrupting pesticides. Reprod Toxicol. 34:237-250; 2012

Lafuente, A.; Pereiro, N. Neurotoxic effects induced by endosulfan exposure during pregnancy and lactation in female and male rat striatum. Toxicology. 311:35-40; 2013

Levin, E.D.; Addy, N.; Baruah, A.; Elias, A.; Christopher, N.C.; Seidler, F.J.; Slotkin, T.A. Prenatal chlorpyrifos exposure in rats causes persistent behavioral alterations. Neurotoxicol Teratol. 24:733-741; 2002

Levin, E.D.; Timofeeva, O.A.; Yang, L.; Petro, A.; Ryde, I.T.; Wrench, N.; Seidler, F.J.; Slotkin, T.A. Early postnatal parathion exposure in rats causes sex-selective cognitive impairment and neurotransmitter defects which emerge in aging. Behav Brain Res. 208:319-327; 2010

Lezak, M.D. Neuropsychological Assessment. Oxford: Oxford University Press 1995

Llop, S.; Julvez, J.; Fernandez-Somoano, A.; Santa Marina, L.; Vizcaino, E.; Iniguez, C.; Lertxundi, N.; Gascon, M.; Rebagliato, M.; Ballester, F. Prenatal and postnatal insecticide use and infant neuropsychological development in a multicenter birth cohort study. Environ Int. 59:175-182; 2013

Marinovich, M.; Guizzetti, M.; Ghilardi, F.; Viviani, B.; Corsini, E.; Galli, C.L. Thyroid peroxidase as toxicity target for dithiocarbamates. Archives of Toxicology. 71:508-512; 1997

Marks, A.R.; Harley, K.; Bradman, A.; Kogut, K.; Barr, D.B.; Johnson, C.; Calderon, N.; Eskenazi, B. Organophosphate pesticide exposure and attention in young Mexican-American children: the CHAMACOS study. Environ Health Perspect. 118:1768-1774; 2010

McCarthy, M.M.; Nugent, B.M. Epigenetic contributions to hormonally-mediated sexual differentiation of the brain. Journal of neuroendocrinology. 25:1133-1140; 2013 
Murata, K.; Weihe, P.; Budtz-Jorgensen, E.; Jorgensen, P.J.; Grandjean, P. Delayed brainstem auditory evoked potential latencies in 14-year-old children exposed to methylmercury. The Journal of pediatrics. 144:177-183; 2004

Orton, F.; Rosivatz, E.; Scholze, M.; Kortenkamp, A. Widely Used Pesticides with Previously Unknown Endocrine Activity Revealed as in Vitro Anti-Androgens. Environ Health Perspect. 119:794-800; 2011

Panzica, G.C.; Bo, E.; Martini, M.A.; Miceli, D.; Mura, E.; Viglietti-Panzica, C.; Gotti, S. Neuropeptides and enzymes are targets for the action of endocrine disrupting chemicals in the vertebrate brain. Journal of toxicology and environmental health Part B, Critical reviews. $14: 449-472 ; 2011$

Rauh, V.; Arunajadai, S.; Horton, M.; Perera, F.; Hoepner, L.; Barr, D.B.; Whyatt, R. 7-Year Neurodevelopmental Scores and Prenatal Exposure to Chlorpyrifos, a Common Agricultural Pesticide. Environ Health Perspect; 2011

Rauh, V.A.; Garfinkel, R.; Perera, F.P.; Andrews, H.F.; Hoepner, L.; Barr, D.B.; Whitehead, R.; Tang, D.; Whyatt, R.W. Impact of prenatal chlorpyrifos exposure on neurodevelopment in the first 3 years of life among inner-city children. Pediatrics. 118:e1845-e1859; 2006

Rauh, V.A.; Perera, F.P.; Horton, M.K.; Whyatt, R.M.; Bansal, R.; Hao, X.; Liu, J.; Barr, D.B.; Slotkin, T.A.; Peterson, B.S. Brain anomalies in children exposed prenatally to a common organophosphate pesticide. Proceedings of the National Academy of Sciences of the United States of America. 109:7871-7876; 2012

Raven, J.C.; Court, J.H.; Raven, J. Manual for Raven's Progressive Matrices and vocabulary scales. London: H.K. Lewis; 1979

Rice, D.; Barone, S., Jr. Critical periods of vulnerability for the developing nervous system: evidence from humans and animal models. Environ Health Perspect. 108 Suppl 3:511-533; 2000

Schantz, S.L.; Widholm, J.J. Cognitive effects of endocrine-disrupting chemicals in animals. Environ Health Perspect. 109:1197-1206; 2001

Slotkin, T.A. Cholinergic systems in brain development and disruption by neurotoxicants: nicotine, environmental tobacco smoke, organophosphates. Toxicology and applied pharmacology. 198:132-151; 2004

Swan, S.H.; Liu, F.; Hines, M.; Kruse, R.L.; Wang, C.; Redmon, J.B.; Sparks, A.; Weiss, B. Prenatal phthalate exposure and reduced masculine play in boys. Int J Androl. 33:259-269; 2010

Thorndike, R.L.; Hagen, E.P. Stanford Binet Intelligence Scale. Chicago: Riverside; 1986

Valerius, S.; Sparfeldt, J.R. Consistent g- as well as consistent verbal-, numerical- and figuralfactors in nested factor models? Confirmatory factor analyses using three test batteries. Intelligence. 44:120-133; 2014

Venerosi, A.; Ricceri, L.; Tait, S.; Calamandrei, G. Sex dimorphic behaviors as markers of neuroendocrine disruption by environmental chemicals: the case of chlorpyrifos. Neurotoxicology. 33:1420-1426; 2012

Vinggaard, A.M.; Christiansen, S.; Laier, P.; Poulsen, M.E.; Breinholt, V.; Jarfelt, K.; Jacobsen, H.; Dalgaard, M.; Nellemann, C.; Hass, U. Perinatal exposure to the fungicide prochloraz feminizes the male rat offspring. Toxicological sciences, 85:886-897; 2005

Wechsler, D. Manual for the Wechsler Intelligence Scale for Children-Revised. New York: The Psychological Corporation; 1974

Weiss, B. Same sex, no sex, and unaware sex in neurotoxicology. Neurotoxicology. 32:509-517; 2011 
White, R.F.; Debes, F.; Dahl, R.; Grandjean, P. Development and field testing of a neuropsychological test battery to assess the effects of methylmercury exposure in the Faroe Islands. Japan: Proceedings of the international symposium on assessment of environmental pollution and health effects of methylmercury, Kumamoto; 1994

Winneke, G.; Ranft, U.; Wittsiepe, J.; Kasper-Sonnenberg, M.; Furst, P.; Kramer, U.; Seitner, G.; Wilhelm, M. Behavioral Sexual Dimorphism in School-Age Children and Early Developmental Exposure to Dioxins and PCBs: A Follow-Up Study of the Duisburg Cohort. Environ Health Perspect. 122:292-298; 2014

Wohlfahrt-Veje, C.; Andersen, H.R.; Jensen, T.K.; Grandjean, P.; Skakkebaek, N.E.; Main, K.M. Smaller genitals at school age in boys whose mothers were exposed to non-persistent pesticides in early pregnancy. Int J Androl. 35:265-272; 2012a

Wohlfahrt-Veje, C.; Andersen, H.R.; Schmidt, I.M.; Aksglaede, L.; Sorensen, K.; Juul, A.; Jensen, T.K.; Grandjean, P.; Skakkebaek, N.E.; Main, K.M. Early breast development in girls after prenatal exposure to non-persistent pesticides. Int J Androl. 35:273-282; 2012b

Wohlfahrt-Veje, C.; Main, K.M.; Schmidt, I.M.; Boas, M.; Jensen, T.K.; Grandjean, P.; Skakkebaek, N.E.; Andersen, H.R. Lower birth weight and increased body fat at school age in children prenatally exposed to modern pesticides: a prospective study. Environ Health. $10: 79 ; 2011$ 
Table 1. Characteristics ${ }^{\mathrm{a}}$ of 177 children without (Unexposed) or with (Exposed) prenatal pesticide exposure

\begin{tabular}{|l|l|l|l|}
\hline Parameter & Unexposed (N=65) & Exposed (N=112) & $p$-value \\
\hline Socio-demographic characteristics & & & \\
\hline Parental social class [N (\%)] & & & 0.02 \\
\hline $1-3$ & $26(40.0)$ & $23(20.5)$ & \\
\hline 4 & $25(38.5)$ & $63(56.3)$ & \\
\hline 5 & $14(21.5)$ & $26(23.2)$ & \\
\hline Siblings, yes/no [no. (\%)] & $64(98.5)$ & $108(96.4)$ & 0.65 \\
\hline Number of children in the home & $2.0(2.0 ; 4.0)$ & $2.0(1.6 ; 4.0)$ & 0.23 \\
\hline Maternal smoking in pregnancy [no. (\%)] & $15(23.4)$ & $31(27.7)$ & 0.60 \\
\hline $\begin{array}{l}\text { Maternal alcohol consumption in } \\
\text { pregnancy [no. (\%)] }\end{array}$ & $21(32.8)$ & $42(38.2)$ & 0.62 \\
\hline Mother of non-Danish origin [no. (\%)] & $4(6.2)$ & & 0.47 \\
\hline Children's characteristics & & $4(3.6)$ & \\
\hline No of girls [no. (\%)] & $30(46.2)$ & $53(47.3)$ & 1.00 \\
\hline Birth weight (g) & $3670(2845 ; 4635)$ & $3500(2255 ; 4300)$ & 0.12 \\
\hline Gestational age (days) & $282(262 ; 295)$ & $281(252 ; 297)$ & 0.99 \\
\hline Head circumference at birth (cm) & $36.0(33.0 ; 38.0)$ & $35.0(32.6 ; 38.0)$ & 0.26 \\
\hline $\begin{array}{l}\text { Breastfeeding (exclusively/mainly) [no. } \\
(\%){ }^{\text {c }}\end{array}$ & $15(71.4)$ & $78(70.9)$ & 1.00 \\
\hline Age at examination (years) & & & 0.91 \\
\hline Height (cm) & $8.3(6.5 ; 10.6)$ & $8.6(6.9 ; 10.2)$ & 0.91 \\
\hline Weight (kg) & $134.5(116.2 ; 145.2)$ & $134.5(119.3 ; 145.3)$ & 0.68 \\
\hline Systolic blood pressure (mmHg) & $28.5(20.7 ; 39.9)$ & $30.0(22.8 ; 43.6)$ & 0.51 \\
\hline Diastolic blood pressure (mmHg) & $100.0(88.1 ; 115.7)$ & $100.8(89.2 ; 113.2)$ & 0.55 \\
\hline Permanent hearing loss [no. (\%)] & $57.3(49.1 ; 72.9)$ & $59.0(48.5 ; 69.4)$ & 0.38 \\
\hline Impaired vision [no. (\%)] & $2(3.1)$ & $8(7.2)$ & 0.33 \\
\hline Diagnosed neurological disease [no. (\%)] & $5(7.7)$ & $14(12.6)$ & 0.45 \\
\hline Brain concussion [no. (\%)] & $13(20.0)$ & $4(3.6)$ & 0.71 \\
\hline & & $9(8.0)$ & 0.03 \\
\hline
\end{tabular}

${ }^{a}$ For continuous variables, data represent median (5;95 percentiles). ${ }^{b}$ Differences between groups were tested with Mann-Whitney U-Test (continuous data) or Fishers Exact Test (categorical data with two categories) or Likelihood Ratio (categorical data with more than two categories), ${ }^{\mathrm{c}}$ valid information for 131 children (no information for 44 new controls). 
Table 2. Results for brainstem evoked potential latencies (BAEP) at $40 \mathrm{~Hz}$ and regression coefficients $(\beta)$ with $95 \%$ confidence intervals $(95 \% \mathrm{CI})$ for prenatal pesticide exposure. $\beta$ express the mean difference $(95 \% \mathrm{CI})$ from unexposed children

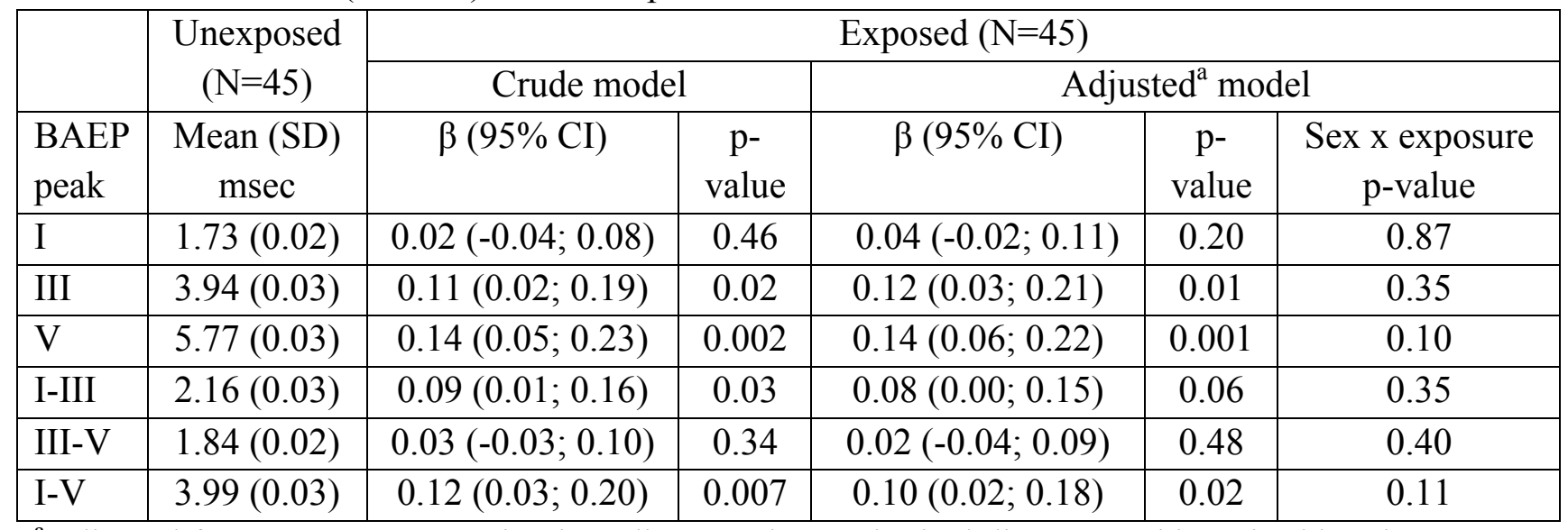

${ }^{\mathrm{a}}$ adjusted for sex, age at examination, diagnosed neurological disease, and impaired hearing. Interaction $\mathrm{p}$-values are from models that include a product term of prenatal exposure and sex and with adjustment for the same covariates. 
Table 3. Adjusted ${ }^{\text {a }}$ results of neuropsychological tests in 6-11 years old children showing mean test scores with 95\% confidence intervals $(95 \% \mathrm{CI})$ for unexposed children and mean score differences $(95 \% \mathrm{CI})$ between prenatally exposed and unexposed children.

\begin{tabular}{|c|c|c|c|c|c|}
\hline \multirow[t]{2}{*}{ Test } & \multirow[b]{2}{*}{$\begin{array}{c}\mathrm{N}_{\text {exposed }} / \\
\mathrm{N}_{\text {unexposed }}\end{array}$} & \multirow{2}{*}{$\begin{array}{l}\text { Unexposed children } \\
\text { Mean test score }(95 \% \\
\mathrm{CI})\end{array}$} & \multicolumn{2}{|c|}{ Exposed children } & \multirow{2}{*}{\begin{tabular}{|c|}
$\begin{array}{c}\text { Sex } x \\
\text { exposure }\end{array}$ \\
p-value
\end{tabular}} \\
\hline & & & $\begin{array}{c}\text { Mean score } \\
\text { difference }(95 \% \mathrm{CI})\end{array}$ & $\begin{array}{c}\mathrm{p}- \\
\text { value }\end{array}$ & \\
\hline \multicolumn{6}{|l|}{ Finger tapping (mean of number of taps in $15 \mathrm{~s}$ ) } \\
\hline Preferred hand & $108 / 63$ & $52.36(50.61 ; 54.12)$ & $-0.95(-3.19 ; 1.29)$ & 0.40 & 0.10 \\
\hline Non-preferred hand & $108 / 63$ & $46.64(44.96 ; 48.34)$ & $-1.07(-3.23 ; 1.09)$ & 0.33 & 0.12 \\
\hline Both hands & $108 / 63$ & $49.51(4.98 ; 51.03)$ & $-1.01(-2.96 ; 0.94)$ & 0.31 & 0.07 \\
\hline Catsys Reaction time (ms), average both hands in $60 \mathrm{~s}$ & $104 / 63$ & $293.6(283.2 ; 304.1)$ & $7.3(-6.2 ; 20.8)$ & 0.29 & 0.27 \\
\hline \multicolumn{6}{|l|}{ CPT II } \\
\hline Reaction time (ms), average for 15 minutes & $105 / 62$ & $422.0(407.3 ; 436.7)$ & $-2.7(-21.5 ; 16.1)$ & 0.78 & 0.28 \\
\hline No. of omission errors & $105 / 62$ & $12.92(9.75 ; 16.10)$ & $1.55(-2.53 ; 5.63)$ & 0.47 & 0.83 \\
\hline No of commission errors & $105 / 62$ & $27.17(25.88 ; 28.46)$ & $1.05(-0.62 ; 2.71)$ & 0.22 & 0.32 \\
\hline Raven coloured progressive matrices, total score $(\mathrm{A}+\mathrm{B})$ & $109 / 63$ & $17.95(17.25 ; 18.65)$ & $-0.18(-1.07 ; 0.71)$ & 0.70 & 0.43 \\
\hline Stanford-Binet, Copying (design 13-28), total score & $109 / 64$ & $22.22(21.53 ; 22.90)$ & $0.41(-0.47 ; 1.28)$ & 0.36 & 0.67 \\
\hline Stanford-Binet Copying 20 minutes recall & $107 / 63$ & $5.36(4.91 ; 5.81)$ & $-0.26(-0.83 ; 0.32)$ & 0.38 & 0.21 \\
\hline WJ-III Picture Vocabulary 60 minutes recall, no cues & $108 / 61$ & $4.61(4.03 ; 5.20)$ & $-0.52(-1.26 ; 0.23)$ & 0.17 & 0.32 \\
\hline Stanford-Binet, sentence repetition & $108 / 63$ & $20.75(19.77 ; 21.74)$ & $-0.40(-1.65 ; 0.85)$ & 0.53 & 0.14 \\
\hline WISC-R digit span forward and backward & $108 / 63$ & $8.72(8.10 ; 9.34)$ & $0.10(-0.70 ; 0.89)$ & 0.81 & 0.18 \\
\hline WJ III Picture Vocabulary, no cue & $109 / 64$ & $14.50(14.22 ; 14.78)$ & $-0.25(-0.60 ; 0.10)$ & 0.16 & 0.05 \\
\hline WJ III Picture Vocabulary, with cues & $109 / 64$ & $15.66(15.43 ; 15.89)$ & $-0.23(-0.52 ; 0.07)$ & 0.13 & 0.05 \\
\hline WJ III Verbal comprehension factor & $108 / 62$ & $0.06(-0.12 ; 0.24)$ & $-0.07(-0.30 ; 0.16)$ & 0.54 & 0.05 \\
\hline
\end{tabular}

adjusted for sex, age (in years) at examination, maternal alcohol consumption in pregnancy (yes or no), social class, history of concussion, diagnosed neurological disease, and for language test (Picture Vocabulary and Verbal comprehension factor) also maternal non-Danish ethnicity (yes or no). Interaction p-values are from models that, besides the variable for prenatal exposure (yes or no), include a product term of prenatal exposure and sex, with adjustment for the same covariates. 
SEM with a Bi-factor Measurement Model

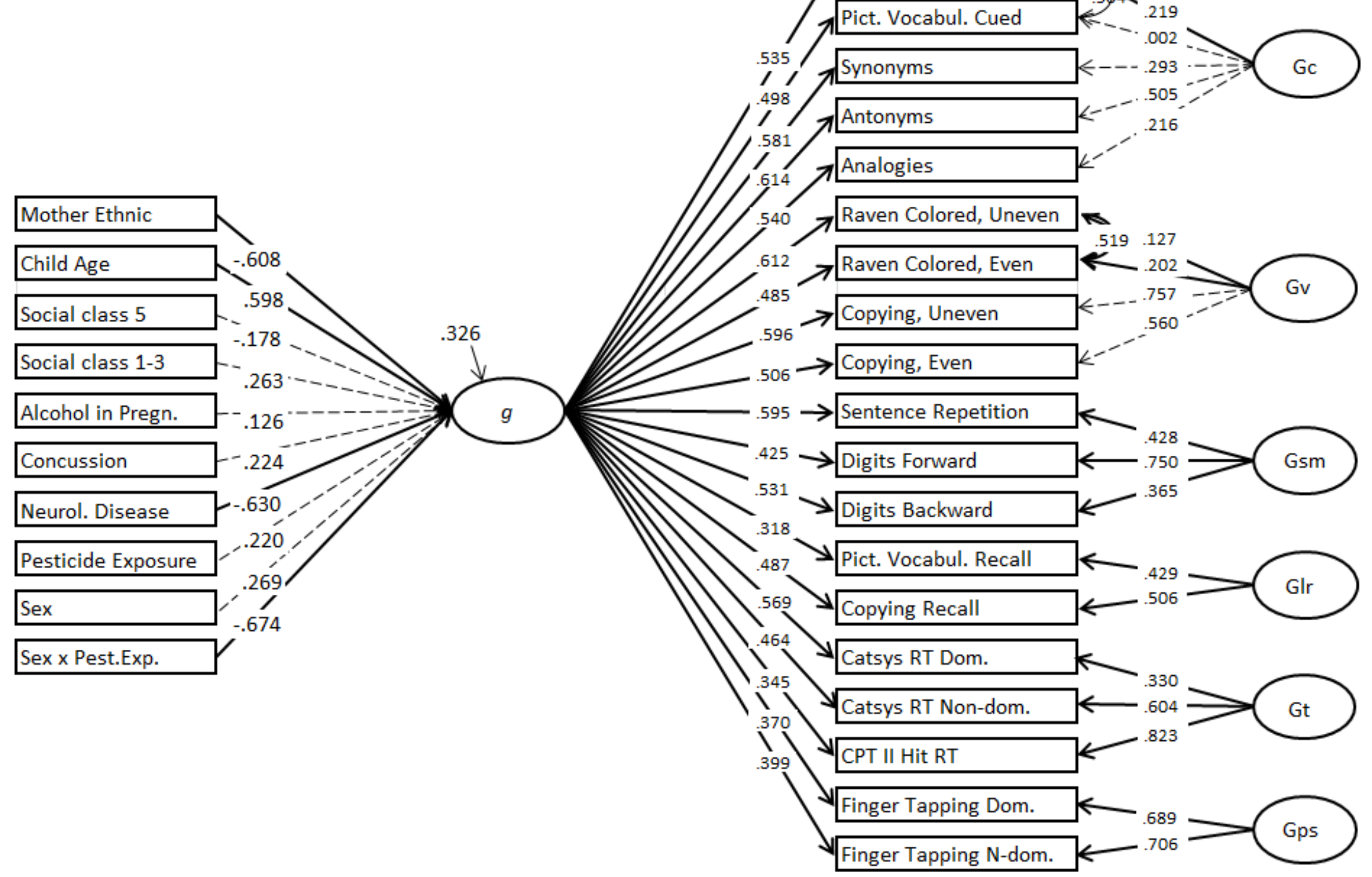


Figure 1. Diagram showing the structural equation model (SEM) used to analyze the interaction effect of sex and prenatal pesticide exposure on general intellectual ability, expressed by the latent variable $g$. The SEM comprises (1) a bi-factor confirmatory factor analytic measurement model specifying a latent variable for general mental ability directly reflected in all the manifest test variables together with six residualized orthogonal broad latent cognitive abilities, also orthogonal with $g$, each reflected in a number of manifest test variables as indicators of that particular intellectual, perceptual or response modality, and (2) a structural model of covariates specifying the changes in the estimated latent variable for general mental ability, commonly referred to as the g-factor, designating a theoretical construct of general intelligence. The residualized factors for the six broad functional domains are verbal comprehension (Gc, crystalized intelligence), visualspatial processing (Gv), short-term memory (Gsm), long-term storage and retrieval (Glr), attention (Gt, reaction and decision speed, chronometrically measured), and psycho-motor speed (Gps). The covariates are the same as those included in the individual regression models were included in the model: sex, age (in years) at examination, maternal alcohol consumption in pregnancy (yes or no), social class, history of concussion, diagnosed neurological disease and maternal non-Danish ethnicity (yes/no). The numbers are standardized estimates of the strength of the linear relations between the different components. Significant relations are indicated by thick arrows, and nonsignificant by dashed arrows. The effect of the only continuous independent variable (Child Age) shows the change in the dependent variable, $g$, in $g$ standard deviation units for a standard deviation change in the independent variable (Child Age). Effects of binary independent variables (all the other predictors) show the change in the dependent variable, $g$, in $g$ standard deviation units when the independent variable changes from zero to one. All independent variables are intercorrelated (paths and coefficients not shown). The proportion of unexplained variance (error variance) in the dependent latent variable, $g$, is indicated by an oblique arrow. Correlations between unexplained variances in manifest variables are specified in terms of local dependency, as indicated by double headed arrows. Raven Colored Progressive Matrices was split into two scales, one for uneven and one for even items, and because of the very close similarity of the stimulus material in both of these scales, as compared to the other scales serving as indicators for the latent variable, Gv, they were considered to be locally dependent. Apart from Picture Vocabulary, no other local dependencies were considered in the bi-factor model. A restriction was put on the two indicators of Glr (Picture Vocabulary Recall and Copying Recall) and the two indicators of Gps (Finger Tapping Dominant and Non-dominant) to have fixed unstandardized coefficients of one. The fit of the estimated measurement model alone was excellent with Chi Square 159.295, df 133, P = 0.0597; RMSEA 0.033 (90\% CI: 0.000 - 0.051); Probability RMSEA $\leq .0$ : 5 0.934; CFI 0.977; TLI 0.971; SRMR $=0.057$. The fit of the SEM with $g$ regressed on the covariates was considered good with Chi Square $=448.996 ; \mathrm{DF}=313 ; \mathrm{P}=0.0000 ; \mathrm{RMSEA}=0.05 ; 90 \% \mathrm{CI}: 0.039-0.06$; Probability RMSEA $\leq .05=0.492 ; \mathrm{CFI}=0.900 ; \mathrm{TLI}=$ $0.885 ; \mathrm{SRMR}=0.073 .52 \%$ of the variance in the manifest variables is accounted for by the g-factor in the bi-factor measurement model and $51 \%$ in the bi-factor SEM. 


\section{Supplementary Tables}

Table A1. Sex-specific adjusted ${ }^{\mathrm{a}}$ mean differences of neuropsychological test scores between prenatally pesticide exposed and unexposed children

\begin{tabular}{|c|c|c|c|c|}
\hline & \multicolumn{2}{|c|}{ Boys (59 exposed, 35 unexposed) } & \multicolumn{2}{|c|}{ Girls (53 exposed, 30 unexposed) } \\
\hline & Score difference $(95 \% \mathrm{CI})$ & p-value & Score difference $(95 \% \mathrm{CI})$ & p-value \\
\hline \multicolumn{5}{|l|}{ Finger tapping (mean of number of taps in $15 \mathrm{~s}$ ) } \\
\hline Preferred hand & $0.73(-2.27 ; 3.73)$ & 0.63 & $-2.90(-6.12 ; 0.32)$ & 0.08 \\
\hline Non-preferred hand & $0.48(-2.41 ; 3.38)$ & 0.74 & $-2.87(-5.98 ; 0.24)$ & 0.07 \\
\hline Both hands & $0.61(-2.00 ; 3.21)$ & 0.65 & $-2.89(-5.68 ;-0.09)$ & 0.04 \\
\hline Catsys, reaction time $(\mathrm{ms})$, average both hands in $60 \mathrm{~s}$ & $0.70(-17.31 ; 18.70)$ & 0.94 & $15.43(-4.14 ; 34.99)$ & 0.12 \\
\hline \multicolumn{5}{|l|}{ CPT II } \\
\hline Reaction time (ms), average for 15 minutes & $-12.25(-37.95 ; 13.16)$ & 0.34 & $7.80(-19.30 ; 34.89)$ & 0.57 \\
\hline Variability & $2.22(-5.16 ; 9.56)$ & 0.55 & $0.04(-7.83 ; 7.91)$ & 0.99 \\
\hline No. of omission errors & $1.90(-3.62 ; 7.42)$ & 0.50 & $1.05(-4.84 ; 6.94)$ & 0.73 \\
\hline No of commission errors & $0.31(-1.94 ; 2.56)$ & 0.79 & $1.94(-0.46 ; 4.33)$ & 0.11 \\
\hline Raven coloured progressive matrices, total score $(\mathrm{A}+\mathrm{B})$ & $0.15(-1.06 ; 1.35)$ & 0.81 & $-0.55(-1.83 ; 0.74)$ & 0.40 \\
\hline Stanford-Binet, Copying (design 13-28), total score & $0.59(-0.59 ; 1.78)$ & 0.32 & $0.22(-1.06 ; 1.50)$ & 0.74 \\
\hline Stanford-Binet Copying 20 minutes recall & $0.09(-0.69 ; 0.86)$ & 0.83 & $-0.64(-1.47 ; 0.19)$ & 0.13 \\
\hline WJ-III Picture Vocabulary 60 minutes recall, no cues & $-0.18(-1.18 ; 0.82)$ & 0.72 & $-0.91(-1.98 ; 0.17)$ & 0.10 \\
\hline Stanford-Binet, sentence repetition & $0.46(-1.22 ; 2.14)$ & 0.59 & $-1.39(-3.19 ; 0.42)$ & 0.13 \\
\hline WISC-R digit span forward and backward & $0.59(-0.48 ; 1.65)$ & 0.28 & $-0.46(-1.60 ; 0.68)$ & 0.42 \\
\hline WJ III Picture Vocabulary, no cue & $0.08(-0.40 ; 0.55)$ & 0.75 & $-0.62(-1.12 ;-0.11)$ & 0.02 \\
\hline WJ III Picture Vocabulary, with cues & $0.04(-0.35 ; 0.53)$ & 0.85 & $-0.54(-0.96 ;-0.12)$ & 0.01 \\
\hline WJ III Verbal comprehension factor & $0.14(-0.17 ; 0.45)$ & 0.38 & $-0.31(-0.64 ; 0.02)$ & 0.07 \\
\hline
\end{tabular}

${ }^{\mathrm{a}}$ Score differences (beta coefficients) obtained from models that, besides the variable for prenatal exposure (yes or no) include a product term of prenatal exposure and sex, and with adjustment for sex, age (in years) at examination, maternal alcohol consumption in pregnancy (yes or no), social class, history of concussion, diagnosed neurological disease, and for language test (Picture Vocabulary and Verbal comprehension factor) also maternal non-Danish ethnicity (yes or no). The corresponding p-values for interaction are presented in Table 3. 
Table A2. Sex-specific adjusted ${ }^{\mathrm{a}}$ mean differences of neuropsychological test scores between prenatally pesticide exposed and unexposed children including only unexposed control children $(\mathrm{N}=21)$ from the original cohort of 133 children

\begin{tabular}{|c|c|c|c|c|c|}
\hline & \multicolumn{2}{|c|}{$\begin{array}{l}\text { Boys (59 exposed, } 14 \\
\text { unexposed) }\end{array}$} & \multicolumn{2}{|c|}{$\begin{array}{l}\text { Girls (53 exposed, } 7 \\
\text { unexposed) }\end{array}$} & \multirow{2}{*}{$\begin{array}{c}\text { Sex x exposure } \\
\text { p-value }\end{array}$} \\
\hline & $\begin{array}{l}\text { Score difference } \\
\qquad(95 \% \mathrm{CI})\end{array}$ & $\begin{array}{c}\mathrm{p}- \\
\text { value }\end{array}$ & $\begin{array}{l}\text { Score difference }(95 \% \\
\mathrm{CI})\end{array}$ & $\begin{array}{c}\mathrm{p}- \\
\text { value }\end{array}$ & \\
\hline \multicolumn{6}{|l|}{ Finger tapping (mean of number of taps in $15 \mathrm{~s}$ ) } \\
\hline Preferred hand & $1.48(-2.70 ; 5.67)$ & 0.48 & $-4.05(-9.60 ; 1.51)$ & 0.15 & 0.12 \\
\hline Non-preferred hand & $-0.31(-4.24 ; 3.63)$ & 0.88 & $-3.58(-8.81 ; 1.65)$ & 0.18 & 0.33 \\
\hline Both hands & $0.59(-2.95 ; 4.12)$ & 0.74 & $-3.81(-8.50 ; 0.88)$ & 0.11 & 0.14 \\
\hline $\begin{array}{l}\text { Catsys, reaction time }(\mathrm{ms}) \\
\text { Average for both hands in } 60 \mathrm{~s}\end{array}$ & $11.11(-14.95 ; 37.16)$ & 0.40 & $-12.26(-45.95 ; 21.44)$ & 0.47 & 0.28 \\
\hline \multicolumn{6}{|l|}{ CPT II } \\
\hline Reaction time (ms), average for 15 minutes & $-7.08(-43.83 ; 29.67)$ & 0.70 & $7.36(-40.34 ; 55.07)$ & 0.76 & 0.64 \\
\hline Variability & $2.82(-7.89 ; 13.52)$ & 0.61 & $2.62(-11.28 ; 16.52)$ & 0.71 & 0.98 \\
\hline No. of omission errors & $2.85(-5.21 ; 10.91)$ & 0.49 & $-0.85(-11.32 ; 9.61)$ & 0.87 & 0.58 \\
\hline No of commission errors & $-0.79(-3.80 ; 2.22)$ & 0.60 & $0.27(-3.64 ; 4.18)$ & 0.89 & 0.67 \\
\hline Raven coloured progressive matrices, total score $(\mathrm{A}+\mathrm{B})$ & $0.33(-1.32 ; 1.99)$ & 0.69 & $-0.21(-2.41 ; 1.98)$ & 0.85 & 0.70 \\
\hline Stanford-Binet, Copying (design 13-28), total score & $1.75(0.12 ; 3.38)$ & 0.04 & $0.56(-1.60 ; 2.72)$ & 0.61 & 0.39 \\
\hline Stanford-Binet Copying 20 minutes recall & $0.29(-0.84 ; 1.42)$ & 0.61 & $-0.13(-1.63 ; 1.37)$ & 0.87 & 0.66 \\
\hline WJ-III Picture Vocabulary 60 minutes recall, no cues & $-0.21(-1.65 ; 1.23)$ & 0.78 & $-1.90(-3.76 ;-0.04)$ & 0.05 & 0.16 \\
\hline Stanford-Binet, sentence repetition & $1.24(-1.00 ; 3.48)$ & 0.28 & $-0.77(-3.75 ; 2.21)$ & 0.61 & 0.29 \\
\hline WISC-R digit span forward and backward & $1.81(0.34 ; 3.27)$ & 0.02 & $-0.40(-2.35 ; 1.55)$ & 0.69 & 0.08 \\
\hline WJ III Picture Vocabulary, no cue & $0.30(-0.31 ; 0.91)$ & 0.34 & $-0.79(-1.60 ; 0.02)$ & 0.06 & 0.04 \\
\hline WJ III Picture Vocabulary, with cues & $0.49(-0.01 ; 0.98)$ & 0.05 & $-0.69(-1.34 ;-0.03)$ & 0.04 & 0.01 \\
\hline WJ III Verbal comprehension factor & $0.41(-0.03 ; 0.84)$ & 0.07 & $-0.51(-1.07 ; 0.05)$ & 0.07 & 0.01 \\
\hline
\end{tabular}

${ }^{a}$ sex-specific score differences (beta coefficients) obtained from models that, besides the variable for prenatal exposure (yes or no) and sex, include a product term of prenatal exposure and sex, and with adjustment sex, age (in years) at examination, maternal alcohol consumption 
in pregnancy (yes or no), social class, history of concussion, diagnosed neurological disease, and for language test (Picture Vocabulary and Verbal comprehension factor) also maternal non-Danish ethnicity (yes or no). 